\title{
Beam-based Feedback for the NLC Linac
}

\author{
L.J. Hendrickson \\ Stanford Linear Accelerator Center, Stanford University, Stanford, California 94309 \\ N. Phinney \\ Stanford Linear Accelerator Center, Stanford University, Stanford, California 94309 \\ A. Seryi \\ Stanford Linear Accelerator Center, Stanford University, Stanford, California 94309 \\ P. Tenenbaum \\ Stanford Linear Accelerator Center, Stanford University, Stanford, California 94309 \\ M. Woodley \\ Stanford Linear Accelerator Center, Stanford University, Stanford, California 94309
}

\begin{abstract}
The NLC linac train-by-train feedback system is designed to stabilize the beam trajectory, but is also a valuable element in the strategy for emittance preservation. New simulations employ improved strategies [1], allowing beam steering to be performed significantly less often than without the feedback system. Additional simulations indicate that the linac feedback can contribute towards successful operation at noisier sites.
\end{abstract}

Contributed to European Particle Accelerator Conference

Lucerne, Switzerland

July 5 - July 9, 2004

*Work supported by Department of Energy contract DE-AC03-76SF00515. 


\title{
BEAM-BASED FEEDBACK FOR THE NLC LINAC *
}

\author{
L.Hendrickson\#, N.Phinney, A.Seryi, P.Tenenbaum, M.Woodley, SLAC, Menlo Park, California, \\ USA
}

\begin{abstract}
The NLC linac train-by-train feedback system is designed to stabilize the beam trajectory, but is also a valuable element in the strategy for emittance preservation. New simulations employ improved strategies [1], allowing beam steering to be performed significantly less often than without the feedback system. Additional simulations indicate that the linac feedback can contribute towards successful operation at noisier sites.
\end{abstract}

\section{OVERVIEW}

The feedback designs for the NLC are based upon systems which were used successfully in the SLC [2], with some improvements. Dipole correctors control the linac beam trajectory, which is measured with beam position monitors (BPMs). The feedback runs at the train repetition frequency of $120 \mathrm{~Hz}$. The time response has been designed using the tools of modern optimal control theory [3]; an exponential response is typical. Simulations are performed using the Matlab-Liar system [4], which includes a full tracking engine. Feedback algorithms are implemented within Matlab scripts [5]. The improved designs were prototyped and tested on the SLAC linac [6].

\section{DESIGN IMPROVEMENTS}

Simulations show that better emittance preservation can be achieved with a carefully chosen layout of the feedback measurement and control devices. The layout is optimized for best performance with ground motion simulations of 30 minutes of ATL-type motion [7]. The results are then analyzed more thoroughly using a full ground motion model [8] with additional component jitter. $15 \mathrm{~nm}$ of random motion is added to the linac quadrupoles. The optimized design includes 5 calculation loops in the $250 \mathrm{GeV}$ linac, with an additional 3 loops when the linac is upgraded to $500 \mathrm{GeV}$. Each feedback loop includes 4 horizontal dipole correctors and 4 vertical dipole correctors, distributed in 2 sets. Each loop uses 16 BPMs, distributed in 4 sets.

Feedback loops communicate beam information to downstream loops using a "cascade" system [9]. This system was used successfully in the SLC, with some limitations. An improvement for NLC is that all downstream loops receive beam information from all upstream loops [1]. With higher intensity operation, wakefields and chromatic effects make the beam transport nonlinear and oscillations propagate differently depending

\footnotetext{
*Work supported by the U.S. Department of Energy under contract number DE-AC03-76SF00515.

${ }^{*} 1 \mathrm{jh} @$ slac.stanford.edu
}

on their point of origin. When a bunch passes off-axis through the accelerating structures, the asymmetric fields induced by the head of the bunch kick the later particles, producing a tilted distribution which remains after the centroid is corrected. In order to correctly model the beam transport, each feedback must receive information from all of the upstream loops to identify the source of the disturbance and avoid overcorrection. The tilt effect also explains the efficacy of the improved BPM and corrector layout on the emittance results.

\section{Results for Moderate-Noise Sites (like SLAC)}

Excellent results, shown in figures 1 and 2, are obtained for moderate-noise sites. With the new simulation for the $250 \mathrm{GeV}$ Linac, there is only $6 \%$ emittance growth after 30 minutes of ground motion. When the same simulation does not include jitter, the growth is less than $1 \%$.

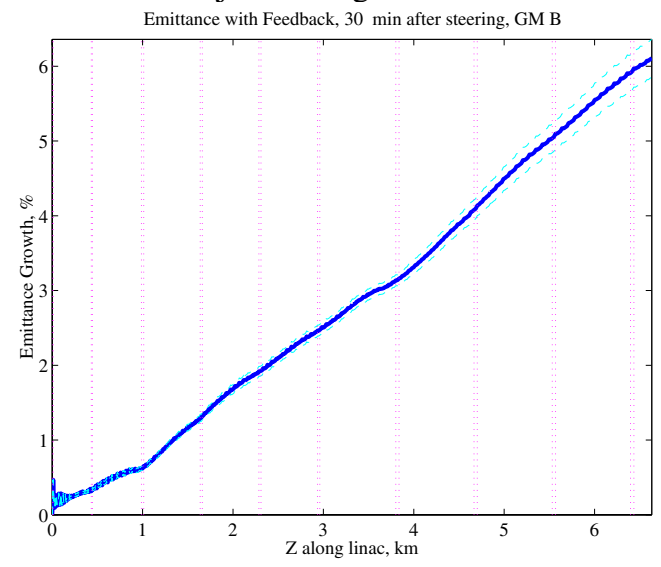

Figure 1: Emittance growth along the linac, after 30 minutes of ground movement with moderate ground motion model (similar to the SLAC linac). Corrector locations shown in vertical dashed lines. Perfect initial steering is assumed (zero emittance growth).

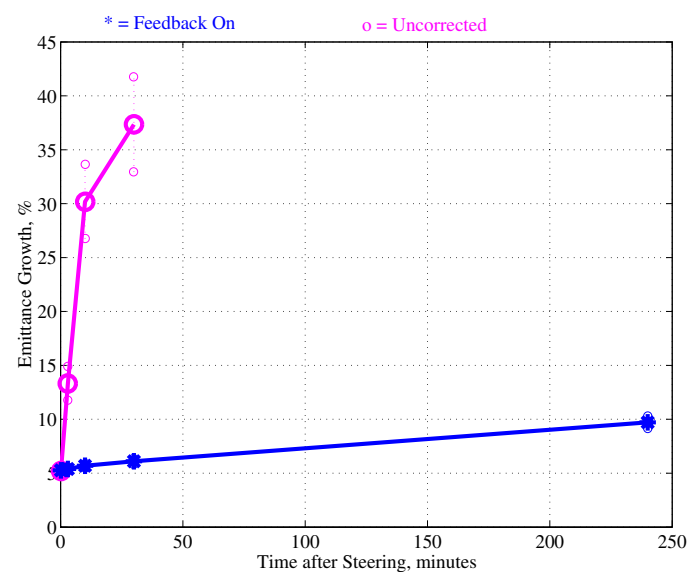

Figure 2: Emittance growth for moderate ground motion, as a function of time after steering. Feedback loops allow the option to preserve emittance $(*)$, while steering less often than without feedback (o) 


\section{Results for Noisier Sites (like KEK)}

For noisier sites such as at KEK, the linac feedback system is particularly helpful in stabilizing the beam, extending the time before steering is required. Without feedback, the emittance grows by $200 \%$ after 3 minutes of ground motion. The feedback system reduces the emittance growth to $8 \%$ after 3 minutes, and $12 \%$ after 10 minutes (figure 3). Note that these simulations assume a perfect initial configuration. More realistically, especially in the presence of beam jitter, the emittance is already degraded. In simulation studies for the $250 \mathrm{GeV}$ linac at a KEK-type site, 2-3 iterations of mover steering bring the emittance growth down to 30\% [11]. This is still within the specified budget of $50 \%$ growth for the linac.

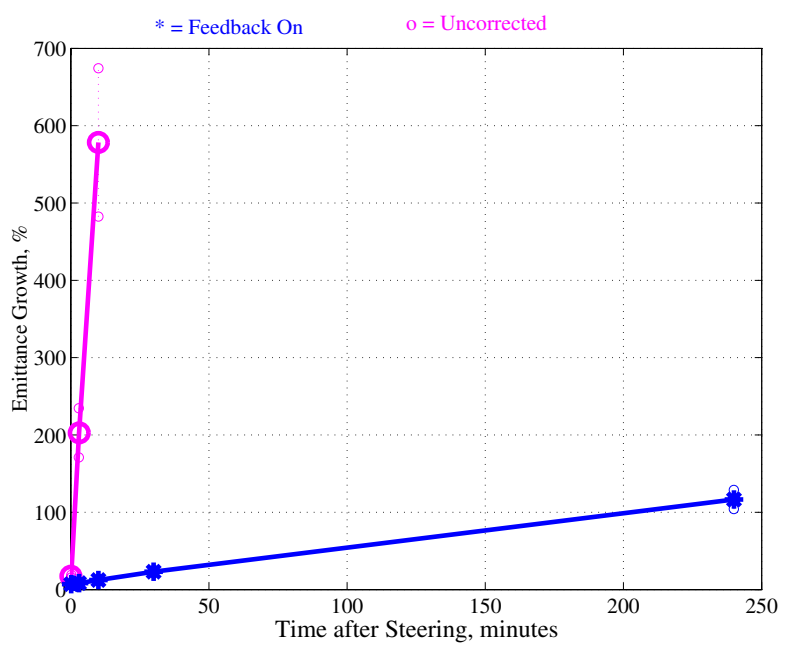

Figure 3: Emittance growth for large ground motion (similar to $\mathrm{KEK}$ ), as a function of time after steering. With the feedback (*), it is possible to wait up to 10 minutes before resteering the beam. Without feedback, the growth (o) quickly becomes excessive.

\section{BPM RESOLUTION SENSITIVITY}

The dependence of feedback performance on BPM resolution was studied for a collider after 30 minutes with modest ground motion. A BPM resolution of 0.2 microns or better is planned for the NLC. Figure 4 shows that the emittance growth is not very sensitive to BPM resolution, and poorer resolution would have minimal impact. Figure 5 shows the resulting beam jitter, which is relatively insensitive to BPM resolution up to as large as 1 micron, for this feedback design. Note that the time response design for the feedback system can also be adjusted to average BPMs over more samples to minimize the impact of poorer BPM resolution.

For most of the feedback simulations reported here, the moderate-noise model is used with a 0.4 micron resolution, while 0.1 microns is used with the noisy-site model.

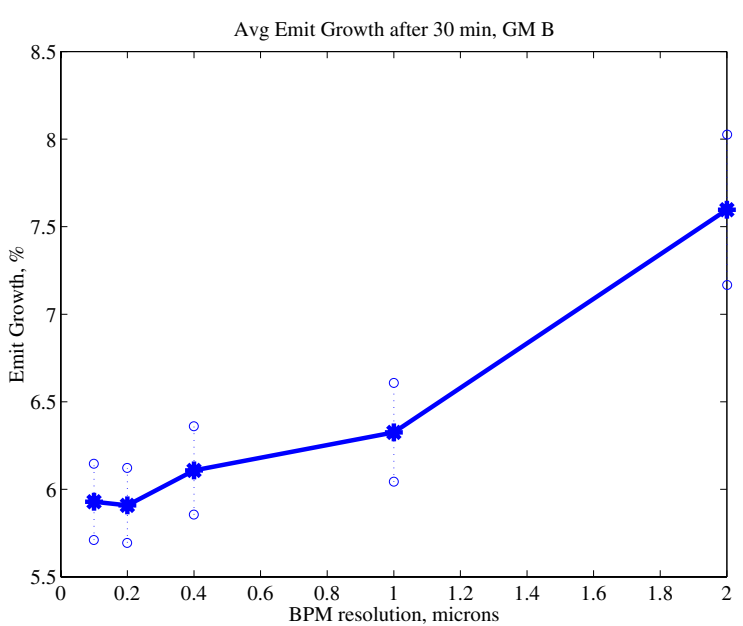

Figure 4: Impact of feedback BPM resolution upon emittance growth.

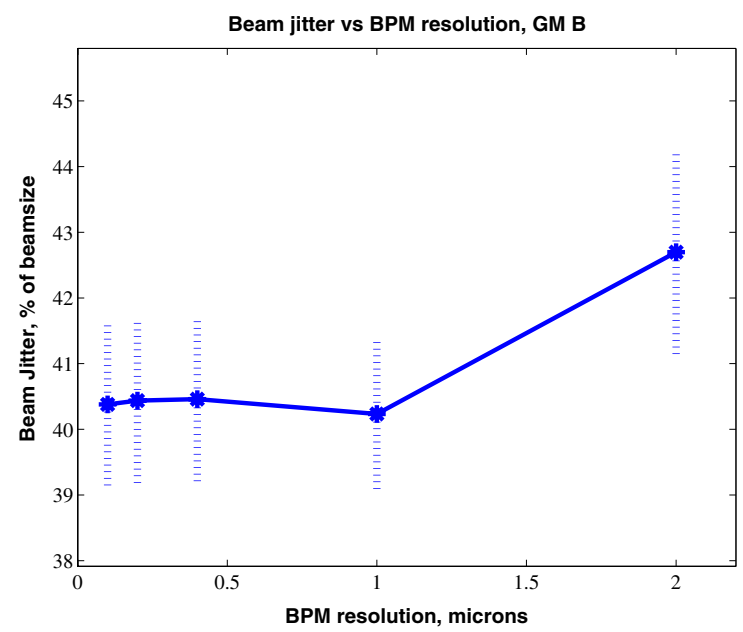

Figure 5: Impact of feedback BPM resolution upon beam jitter.

\section{CORRECTOR SPEED ISSUES}

In the SLC feedback system [2], problems were seen when dipole correctors were slow and poorly modeled. For feedback systems where fast response was needed, high-bandwidth power supplies were used in special sections of the beampipe without copper accelerating structures. Initial NLC simulations assumed very fast corrector response, which could fully implement a correction within a single 120-hz beam pulse, allowing one pulse for system latency [1]. However, this fast correction requires a gap in the accelerator structures, since corrector field changes cannot quickly propagate through the structures to affect the beam. Because a more effective feedback system is obtained with a significant number of correctors, it is desirable to have the option to use slow correctors. The feedback modeling tools have been improved to allow studies of this option. In the simulations for moderate ground motion, we assume that correctors take $8120-\mathrm{Hz}$ pulses to affect the beam. However, to allow for an inaccurate corrector speed estimate, we design an imperfect feedback model which 
underestimates the corrector response by assuming 6120 $\mathrm{Hz}$ pulses for implementation. Figure 6 shows the design response for fast correctors (+), compared to the slow imperfect model (o). Figure 7 shows the design time response to a step function, with the imperfectly modeled slow correctors. Note that the somewhat slow response is partly a design choice for noise characteristics, and partly a result of the slow correctors. An option exists to use modern control techniques to design a feedback which compensates for the slow correctors by overshooting. While this would be useful in theory, in practice it would be very sensitive to timing and latency of the control computers and communications systems; any interruptions to the feedback process might seriously disturb the beam. A more conservative design is chosen that simply models the slow correctors without overcompensating for them.

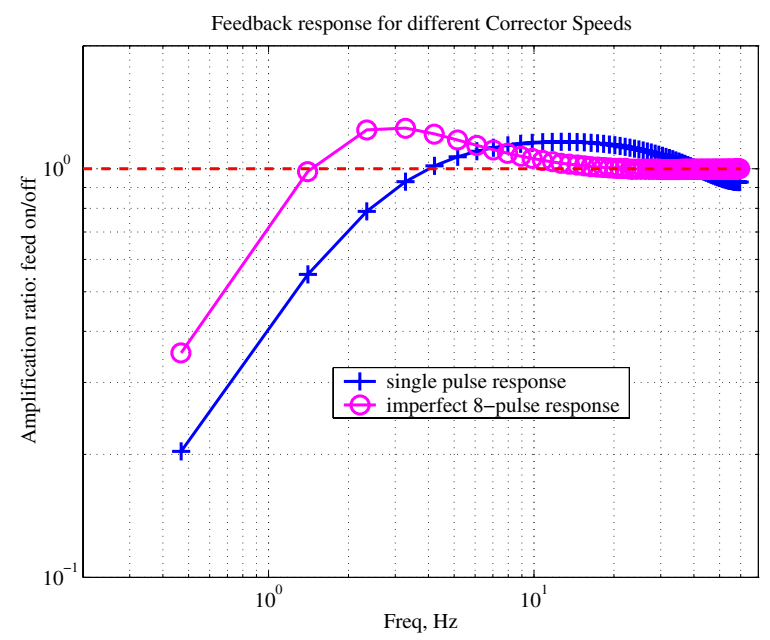

Figure 6: Design response for different corrector speed assumptions. Note that amplification of beam noise occurs when the amplification ratio is above 1. The imperfect slow correctors have poorer response, but due to the built-in averaging, the effect is not substantial.
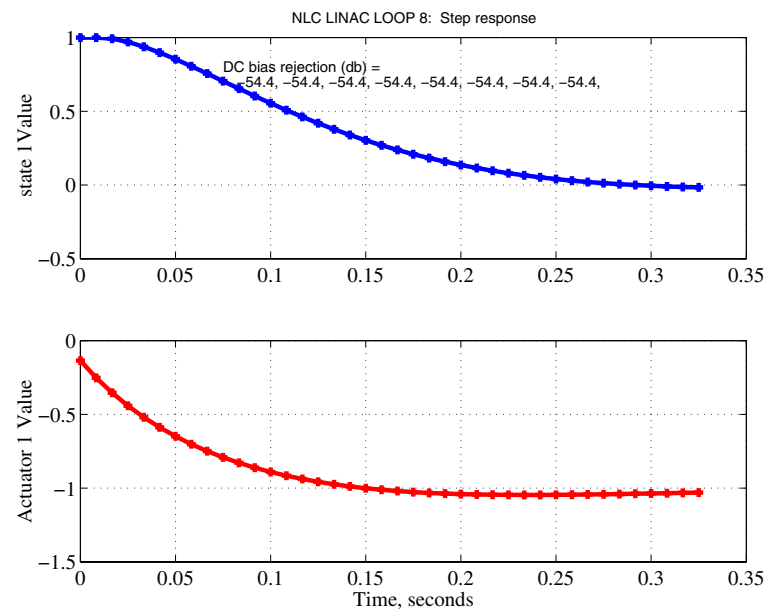

Figure 7: Time response to a step function, for a case in which correctors respond in 8 pulses, but are imperfectly modeled with a 6 pulse response. The top figure shows a controlled feedback state responding, after a sudden step function at time 0 . The lower figure shows a calculated actuator setting for the same timescale; the actuator's effect on the beam is not shown.

\section{CONCLUSIONS}

The feedback system can substantially increase the time allowed between full linac steering. This is especially effective at noisier sites, where the uncorrected emittance degrades to unacceptable levels after only a few minutes. For noisy sites, the feedback systems limits emittance growth to $12 \%$ after 10 minutes of ground motion. For moderate sites, emittance growth is less than $10 \%$, after 4 hours of ground motion.

Work is underway to combine linac feedback simulations with full 2-beam simulations including beam delivery feedback systems. The design time response for the linac system should be reoptimized in order to provide a reasonable operator response, good emittance preservation, and minimal beam jitter.

\section{ACKNOWLEDGEMENTS}

The authors would like to thank T. Raubenheimer and K. Kubo for helpful discussions and results.

\section{REFERENCES}

[1] Beam-based Feedback Simulations for the NLC Linac, L. Hendrickson et al., LINAC, Monterey, California (2000)

[2] L. Hendrickson, et al., "Generalized Fast Feedback System in the SLC," ICALEPCS, Tsukuba, Japan, SLAC-PUB-5683 (1991).

[3] T. Himel, et al., "Use of Digital Control Theory State Space Formalism for Feedback at SLC," PAC, San Francisco, CA, SLAC-PUB-5470 (1991).

[4] P. Tenenbaum, et al., "Use of Simulation Programs for the Modeling of the Next Linear Collider," PAC, New York, New York (1999).

[5] http://www.mathworks.com/

[6] L. Hendrickson, et al., "Beam-based Feedback Testing and Simulations for the SLC Linac," LINAC, Monterey, California (2000).

[7] V. Shiltsev, "Space-Time Ground Diffusion: The ATL Law for Accelerators," Proceedings IWAA-93, 352 (1995).

[8] A Seryi, et al., "Simulation Studies of the NLC with Improved Ground Motion Models," LINAC, Monterey, California (2000).

[9] T. Himel, et al., "Adaptive Cascaded Beam-Based Feedback at the SLC," PAC, Washington, D.C., SLAC-PUB-6125 (1993). [10] A. Seryi, et al., "Vibrational Stability of GLC/NLC Linear Collider: Status and R\&D Plans," in these proceedings.

[11] K. Kubo, email communication, May 21, 2004. 